\title{
Capacidade de exercício e nível de atividade física diária de crianças e adolescentes com fibrose cística: associação com estado nutricional, função pulmonar, hospitalização e uso de antibióticos
}

Exercise capacity and daily physical activity levels in children and adolescents with cystic fibrosis: association with nutritional status, lung function, hospitalization and antibiotic use Capacidad de ejercicio y nivel de actividad física diaria de niños y adolescentes con fibrosis quistica: asociación con estado nutricional, función pulmonar, hospitalización y uso de antibióticos

Leticia Tosts da Silva', Ramon da Silva Pereira², Pamela Vidal'3 , Fernanda Mayrink Gonçalves Liberato ${ }^{4}$, Luana da Silva Baptista Arpini ${ }^{5}$, Roberta Ribeiro Batista Barbosa ${ }^{6}$

RESUMO | O objetivo deste trabalho é avaliar a capacidade de exercício e nível de atividade física diária de crianças e adolescentes com fibrose cística e associar com estado nutricional, função pulmonar, tempo de hospitalização e uso de antibióticos. Trata-se de estudo transversal em indivíduos com fibrose cística entre 6 e 18 anos, registrando-se informações sobre perfil clínico, histórico de hospitalizações e uso de antibióticos. Os participantes foram submetidos à espirometria, bioimpedância, avaliação da capacidade de exercício com teste de Shuttle modificado (MST), o nível da atividade física foi medido por meio do questionário internacional de atividade física (IPAQ) e usando acelerômetro por 5 dias. Participaram 30 indivíduos com idade de 11,2 \pm 3,6 anos e volume expirado forçado $\left(V F_{1}\right)$ de 68,0 $\pm 24,8 \%$. A mediana da distância percorrida no MST foi de 820 metros (66,3\%) e demonstrou associação com a função pulmonar $(r=0,78)$, estado nutricional $(r=0,38)$, tempo de hospitalização $(r=-0,42)$ e uso de antibióticos $(r=-0,46)$. De acordo com o questionário, 20 pacientes (64,6\%) foram classificados como sedentários, o acelerômetro revelou que os indivíduos passam 354,2 minutos em atividades sedentárias e apenas 14,9 minutos em atividades moderadas a vigorosas por dia. Quanto maior a porcentagem de tempo em atividade física moderada a vigorosa, maior o índice de massa corpórea (IMC) e menor o tempo de hospitalização. Assim, a capacidade de exercício e nível de atividade física em crianças e adolescentes com fibrose cística apresentou-se reduzida e associada com menores valores de IMC e aumento do tempo de hospitalização.

Descritores | Fibrose Cística; Capacidade de Exercício; Nível de Atividade Física; Antibióticos; Hospitalização.

ABSTRACT I To evaluate the exercise capacity and daily physical activity level among children and adolescents with cystic fibrosis, and its association with nutritional status, lung function, hospitalization time, and days taking antibiotics. This is a cross-sectional study in individuals with cystic fibrosis aged 6 to 18 years. Information on clinical profile, history of hospitalizations and antibiotic use were collected. Participants were submitted to spirometry, bioimpedance,

'Escola Superior de Ciências da Santa Casa de Misericórdia (Emescam) - Vitória (ES), Brasil. E-mail: robertaribeirob@hotmail.com. ORCID-0000-0001-9348-8364

Escola Superior de Ciências da Santa Casa de Misericórdia (Emescam) - Vitória (ES), Brasil. E-mail: robertaribeirob@hotmail.com. ORCID-0000-0002-2441-7690

${ }^{3}$ Escola Superior de Ciências da Santa Casa de Misericórdia (Emescam) - Vitória (ES), Brasil. E-mail: robertaribeirob@hotmail.com. ${ }^{4}$ Hospital Infantil Nossa Senhora da Glória - Vitória (ES), Brasil. E-mail: robertaribeirob@hotmail.com. ORCID-0000-0001-5227-2022 ${ }_{5}^{5}$ Hospital Infantil Nossa Senhora da Glória - Vitória (ES), Brasil. E-mail: robertaribeirob@hotmail.com. ORCID-0000-0002-7989-6895 ${ }^{6}$ Escola Superior de Ciências da Santa Casa de Misericórdia (Emescam) - Vitória (ES), Brasil. E-mail: robertaribeirob@hotmail.com. ORCID-0000-0001-9283-9097 
and an assessment of exercise capacity with modified shuttle test (MST), and the level of physical activity was measured with the International Physical Activity Questionnaire (IPAQ) using an accelerometer for 5 days. In total, 30 individuals participated, aged $11.2 \pm 3.6$ years, and $68.0 \pm 24.8 \%$ in forced expired volume in the first second $\left(F E V_{1}\right)$. The median distance covered in the MST was 820 meters (66.3\%), showing association with lung function $(r=0.78)$, nutritional status $(r=0.38)$, hospitalization time $(r=-0.42)$ and antibiotic use $(r=-0.46)$. According to the questionnaire, 20 patients (64.6\%) were classified as sedentary, the accelerometer revealed that the individuals spend 354.2 minutes in sedentary activities and only 14.9 minutes in moderate to vigorous activities per day. The higher the percentage of time in moderate to vigorous physical activity, the higher the body mass index (BMI) and the shorter the hospitalization time. Exercise capacity and level of physical activity in children and adolescents with cystic fibrosis is reduced and associated with lower BMI values and with an increase in hospitalization time.

Keywords | Cystic Fibrosis; Exercise Capacity; Physical Activity Level; Antibiotics; Hospitalization.

RESUMEN | El propósito de este trabajo fue evaluar la capacidad de ejercicio y el nivel de actividad física diaria de los niños y adolescentes con fibrosis quística, así como asociarlos con el estado nutricional, la función pulmonar, la duración de la permanencia hospitalaria y el uso de antibióticos. Este es un estudio transversal realizado con individuos con fibrosis quística con edades comprendidas entre los 6 y los 18 años, y que registra información sobre el perfil clínico, los antecedentes de hospitalizaciones y el uso de antibióticos. Los participantes se sometieron a la espirometría, la bioimpedancia, la evaluación de la capacidad de ejercicio con la prueba de Shuttle modificada (MST); para medir el nivel de actividad física se aplicó el Cuestionario Internacional de Actividad Física (IPAQ) y el uso del acelerómetro durante 5 días. Participaron 30 personas de entre 11,2 \pm 3,6 años de edad y volumen espirado forzado $\left(V_{E F}\right)$ de 68,0 $\pm 24,8 \%$. La mediana de la distancia recorrida en el MST fue de 820 metros (66,3\%) y se mostró asociación con la función pulmonar ( $r=0,78)$, el estado nutricional $(r=0,38)$, la duración de la permanencia hospitalaria $(r=-0,42)$ y el uso de antibióticos $(r=-0,46)$. El cuestionario reveló que 20 pacientes (64,6\%) estaban sedentarios, y el acelerómetro evidenció que ellos pasan 354,2 minutos en actividades sedentarias y solo 14,9 minutos en actividades moderadas a intensas al día. Cuanto mayor sea el porcentaje de tiempo dedicado a la actividad física moderada a intensa, mayor será el índice de masa corporal (IMC) y menor la duración de la permanencia hospitalaria. Por lo tanto, la capacidad de ejercicio y el nivel de actividad física de niños y adolescentes con fibrosis quística fueron menores y están asociadas con bajos valores de IMC y con una permanencia hospitalaria más prolongada.

Palabras clave | Fibrosis Quística; Capacidad de Ejercicio; Nivel de Actividad Física; Antibióticos; Hospitalización.

\section{INTRODUÇÃO}

A Fibrose Cística (FC) é uma doença genética autossômica recessiva que afeta diversos sistemas orgânicos, dentre eles, o sistema respiratório, com infecções persistentes, obstrução das vias aéreas e redução progressiva do volume expirado forçado no primeiro segundo $\left(\mathrm{VEF}_{1}\right)$, resultando em dispneia, intolerância ao exercício e estilo de vida sedentário ${ }^{1,2}$. Além disso, o envolvimento do sistema gastrointestinal acarreta déficit nutricional, contribuindo para redução de massa muscular, inatividade física ${ }^{3-5}$, osteopenia, osteoporose e perda funcional ${ }^{6}$.

Dados epidemiológicos apontam que $27 \%$ das crianças e 41\% dos adolescentes com FC sofrem um ou mais episódios de exacerbações respiratórias, necessitando de internação por 10 a 11 dias em média. O período de internação leva ao afastamento do ambiente escolar e familiar, causando prejuízo na qualidade de vida ${ }^{7}$. A quantidade de exacerbações e o tempo inferior a seis meses entre estas, aumentam a morbidade e mortalidade por $\mathrm{FC}^{8}$.
Estudos demonstram que a capacidade aeróbica é preditora para hospitalização e mortalidade em pacientes com FC. A redução da capacidade de exercício tem origem multifatorial, dentre as quais podemos citar a obstrução da via aérea, idade, estado nutricional, força muscular respiratória e periférica ${ }^{9-11}$. Schneiderman-Walker et al. afirmam que a capacidade de exercício exerce influência sobre o aparelho respiratório, melhorando a depuração das vias aéreas e levando ao declínio mais lento da função pulmonar ${ }^{12}$.

Donadio et al. demonstraram que a capacidade de exercício não necessariamente segue o declínio esperado da função pulmonar ao longo do tempo 9 . Ledger et al. encontraram uma redução da necessidade de uso de antibiótico durante o ano de intervenção com exercícios físicos, desempenhando um papel importante no declínio da função pulmonar ${ }^{13}$. Uma revisão sistemática realizada em 2019 demonstrou que indivíduos com FC, com consumo máximo de oxigênio $\left(\mathrm{VO}_{2}\right)<82 \%$ do previsto ou $45 \mathrm{~mL} / \mathrm{kg} / \mathrm{ml}$, tiveram um risco de mortalidade 4,9 vezes maior ${ }^{14}$. 
Dessa forma, a avaliação da capacidade de exercício em pacientes com FC é extremamente importante, sendo um potencial marcador para evolução da doença. O teste de exercício cardiopulmonar é considerado padrão-ouro na avaliação da capacidade de exercício, porém sua aplicação é limitada, uma ótima opção são os testes de campo, como o teste de shuttle modificado (MST), que tem demonstrado melhor sensibilidade e especificidade em indivíduos com $\mathrm{FC}^{15,16}$.

Além disso, estudos apontam que quanto maior o tempo gasto em atividades físicas moderadas diariamente, menor a frequência de exacerbações e necessidade de hospitalização em pacientes com $\mathrm{FC}^{17,18}$. Uma recente revisão sistemática apontou forte associação do nível de atividade física, mensurado de forma objetiva por meio da acelerometria, com desfechos clínicos e com a capacidade de exercício, entretanto, concluiu também que o número de estudos que utilizaram o acelerômetro é limitado e apresentam fragilidades metodológicas ${ }^{19}$.

É importante que sejam realizados estudos com testes de tolerância de exercício e avaliação de nível de atividade física diária utilizando acelerômetro, para melhor compreensão das associações com variáveis clínicas preditoras de prognóstico, melhorar o acompanhamento de pacientes com $\mathrm{FC}$ em centros de referência e direcionar a terapêutica, visando a manutenção da função pulmonar, redução de exacerbações e hospitalizações.

Portanto, o objetivo deste trabalho foi avaliar a capacidade de exercício e nível de atividade física utilizando acelerometria e associando ao estado nutricional, à função pulmonar, ao tempo de hospitalização e ao uso de antibiótico em crianças e adolescentes com fibrose cística.

\section{METODOLOGIA}

Estudo transversal com amostra de pacientes com Fibrose Cística, com idade entre seis e 18 anos, e que receberam acompanhamento no Centro de Referência do Estado do Espírito Santo entre março e dezembro de 2018. Usou-se como critério para a participação deste estudo o diagnóstico clínico confirmado pelo teste do suor ou pelo teste genético, ter condições clínicas estáveis e função cognitiva preservada. Como critérios para a exclusão foram adotadas intercorrências nos últimos 30 dias antes da pesquisa e indivíduos que possuíam dificuldades para realizar os testes propostos. Finalmente, foram elegíveis 47 pacientes. Considerando que o nível de atividade física e a capacidade de exercício são os objetos principais do estudo, para fins de cálculo amostral, utilizou-se como base o estudo de Savi et al. ${ }^{20}$. A partir de desvios padrões estipulados em dois, poder de $95 \%$, índice de significância de 5\%, correlação mínima entre as variáveis de 0,5 , estimou-se um tamanho amostral de 28 indivíduos.

Inicialmente foi entregue, lido e assinado, em duas vias, o termo de consentimento livre e esclarecido e o termo de consentimento livre e esclarecido. Ainda, foram registradas as seguintes informações do prontuário: idade, sexo, escore Shwachman-Kulczycki (SK), presença e tipo de colonização crônica, dias de internação hospitalar e de uso de antibióticos no último ano.

A espirometria foi realizada utilizando aparelho da marca KoKo (nSpire Health, Inc., Circle Longmont, EUA), devidamente calibrado, seguindo os critérios da American Thoracic Society. Foram analisadas as variáveis: capacidade vital forçada $(\mathrm{CVF}) ; \mathrm{VEF}_{1 ;}$ fluxo expirado forçado entre 25 e $75 \%\left(\mathrm{FEF}_{25-75 \%}\right)$; e a relação $\mathrm{VEF}_{1} /$ CVF. Os valores previstos foram obtidos utilizando-se a equação the global lung initiative $2012^{21}$.

Para avaliação do estado nutricional foi utilizada a bioimpedância, com equipamento Inbody 720 (InBody Co., Los Angeles, EUA), que utiliza o método de medição direta segmentar por multifrequência, com eletrodos tetrapolar em oito pontos táteis. Os participantes foram orientados a seguirem algumas recomendações antes do exame, como jejum absoluto de 12 horas, não realizar exercícios físicos extenuantes e não consumir bebidas à base de cafeína nas $24 \mathrm{~h}$ antecedentes e urinar antes da realização do exame. Foram obtidos os dados de peso, massa muscular esquelética, massa de gordura e índice de massa corpórea (IMC).

A seguir, foi realizada a avaliação da capacidade de exercício por meio do teste de esforço máximo, o teste de Shuttle modificado (MST), validado para a população de Fibrose Cística. Para o teste, foi utilizada uma pista de 10 metros, em que o indivíduo foi instruído a caminhar dentro do ritmo determinado por sinais sonoros, até a fadiga ou presença de sintomas limitantes. O teste foi interrompido quando paciente apresentou incapacidade de manter o ritmo ou atingiu a frequência cardíaca máxima. A saturação periférica de oxi-hemoglobina $\left(\mathrm{SpO}_{2}\right)$ e a frequência cardíaca foram registradas ao final de cada nível do teste, utilizando um oxímetro de dedo da marca Nonin, fixado ao dedo do paciente durante todo o teste. Antes e após o teste foram aferidas pressão arterial, 
frequência cardíaca, $\mathrm{SpO}_{2}$ e frequência respiratória. As variáveis fornecidas pelo MST foram a distância máxima percorrida, a velocidade máxima alcançada, pressão arterial, frequência cardíaca, estágio e respectivo percurso no qual o teste foi interrompido. A distância predita foi calculada utilizando a equação proposta no estudo de Lanza et al.

A versão curta do questionário internacional de atividade física (IPAQ) foi utilizada para avaliar o nível de atividade física diária. Para os sujeitos com idade até 12 anos foi utilizado o IPAQ-C e os indivíduos foram classificados em ativos ( $\geq 3$ pontos) ou sedentários $(<3$ pontos). Para os indivíduos com idade superior a 12 anos, foi utilizado o IPAQ-A, e foram classificados como ativos quando havia somatório de pelo menos 150 minutos por semana de atividades físicas de intensidade moderada, subdivididas em no mínimo três vezes/ semana; ou atividades físicas vigorosas por pelo menos três sessões de 20 minutos/semana ${ }^{23}$. Finalmente, os participantes utilizaram um acelerômetro triaxial wGT3X-BT (Vermont, San Sebastian, Espanha), fixado no lado esquerdo da cintura para avaliar de forma objetiva o nível de atividade física diária por cinco dias seguidos. Os resultados foram expressos em média de counts/min, sendo classificado em sedentária $(<100$ counts), leve ( $\geq 100$ counts) e moderada a vigorosa (>2296 counts) $)^{24}$.

A normalidade dos dados foi avaliada pelo teste de Shapiro-Wilk. Os dados contínuos normais foram expressos em média e desvio padrão e os dados assimétricos em mediana e intervalo interquartil. Para correlacionar as variáveis da capacidade de exercício e níveis de atividade física diária com as variáveis clínicas foi utilizada a correlação de Spearman. A interpretação do coeficiente de correlação ( $r$ ) foi: correlação fraca quando $r<0,4$, correlação moderada quando $r>0,4$ e $r<0,7$, e correlação forte quando $r>0,7$. Todas as análises e processamento dos dados foram realizados no programa SPSS 20.0. O nível de significância adotado foi de $5 \%(\mathrm{p}<0,05)$.

\section{RESULTADOS}

Participaram deste estudo 30 crianças e adolescentes com idade de $11,2 \pm 3,6$ anos, a maioria do sexo feminino, IMC de $16,6 \pm 3 \mathrm{~kg} / \mathrm{m}^{2}$, com $\mathrm{VEF}_{1}$ de $68,8 \pm 24,8 \%$ do predito, sendo que $10(33,3 \%)$ tinham função pulmonar grave, o tempo médio de internação hospitalar de $6 \pm 13,9$ dias e de tempo de uso de antibióticos de 33,9 $\pm 26,9$ dias no último ano (Tabela 1).
Tabela 1. Perfil demográfico, antropométrico e clínica da amostra estudada

\begin{tabular}{lr}
\multicolumn{1}{c}{ Variáveis } & \multicolumn{1}{c}{$n=30$} \\
Idade & $11,2 \pm 3,6$ \\
Sexo Feminino (\%) & $19(63,3)$ \\
Raça branca (\%) & $17(56,6)$ \\
Tipo de Mutação (\%) & \\
$\quad$ Heterozigoto Fdel 508 & $14(46,6)$ \\
Colonização (\%) & \\
Staphylococcus aureus & $20(65)$ \\
Escore SK & \\
SK Total* & $86,0 \pm 13,3$ \\
Estado Nutricional & $16,6 \pm 3$ \\
IMC & $-0,74 \pm 1,16$ \\
Escore Z IMC & $13,3 \pm 5$ \\
MME (Kg) & $7,0 \pm 5$ \\
MG (Kg) & \\
Função Pulmonar & $68,0 \pm 24,8$ \\
VEF, & $77,7 \pm 21,8$ \\
CVF\% & $53,0 \pm 30,0$ \\
FEF & $6,-75 \%$ \\
Hospitalização (dias/ano) & $6,0 \pm 13,9$ \\
Antibiótico (dias/ ano) & $33,9 \pm 26,9$
\end{tabular}

Legenda: SK: Shwachman-Kulczycki; IMC: indice de massa corporal; MME: massa muscular esquelética: MG: massa de gordura: VEF\%: volume expirado no primeiro segundo; CVF\%: capacidade vital forçada; $\mathrm{FEF}_{25-75 \%}$ : fluxo expiratório forçado entre $25-75 \%$.

A capacidade de exercício medida pelo MST foi de 820 metros de distância percorrida, correspondendo a $66,3 \pm 23,3 \%$ do valor predito. De acordo com o questionário IPAQ, 20 indivíduos (64,6\%) foram classificados como sedentários. A avaliação do nível de atividade física, por meio do acelerômetro, mostrou que os participantes dedicam cerca de $354,2 \mathrm{~min} / \mathrm{dia}(54,7 \%)$ do tempo diário realizando atividades sedentárias, e apenas $14,9 \mathrm{~min} / \mathrm{dia}(2,4 \%)$ do tempo diário em atividades moderadas e vigorosas. Os dados de capacidade de exercício e nível de atividade física diária estão expressos na Tabela 2. Observamos uma correlação positiva e moderada $(r=0,55)$ entre o tempo de atividade física moderada e vigorosa com a porcentagem da distância percorrida no teste de Shuttle $(\mathrm{p}=0,002)$.

Tabela 2. Capacidade de exercício e nível de atividade física

\begin{tabular}{|c|c|}
\hline Variáveis & $\mathrm{n}=30$ \\
\hline \multicolumn{2}{|c|}{ Teste de Shuttle Modificado - MST } \\
\hline Nível & $10,8 \pm 2$ \\
\hline Distância (metros)* & $820(350-1400)$ \\
\hline Distância predita (\%) & $66,3 \pm 23,3$ \\
\hline \multicolumn{2}{|l|}{ Antes MST } \\
\hline Frequência cardíaca & $99,4 \pm 14$ \\
\hline Frequência respiratória & $24,7 \pm 8$ \\
\hline $\mathrm{SpO}_{2}(\%)$ & $97 \pm 2$ \\
\hline Borg dispneia & $0,3 \pm 1$ \\
\hline
\end{tabular}

(continua) 
Tabela 2. Continuação

\section{Variáveis}

$n=30$

\section{Após MST}

Frequência cardíaca

$190 \pm 15$

Frequência respiratória

$41,7 \pm 10$

$\mathrm{SpO}_{2}(\%)$

$95,9 \pm 5$

Borg dispneia

$6,9 \pm 3$

Acelerômetro $(n=30)$

Sedentário $\left(\mathrm{min} / \mathrm{dia}^{-1}\right)^{*}$

Sedentário (\%)

Atividade leve $\left(\mathrm{min} / \mathrm{dia}^{-1}\right)^{*}$

Atividade leve (\%)

Atividade moderada a vigorosa $\left(\mathrm{min} / \mathrm{dia}^{-1}\right)^{*}$

Atividade moderada a vigorosa (\%)

Questionário de Atividade Física (IPAQ)

Sedentário (\%)

Questionário internacional de atividade física.

*Variáveis assimétricas, expressas em mediana e intervalo interquartil.

$354,2(246,2-488,5)$

$42.9 \pm 15$

$479,7(371,2-561,3)$

$54,7 \pm 13,5$

$14,9(2,1-36,9)$

$2,4 \pm 2,4$

$20(64,6)$

moglobina; IPAQ:
A capacidade de exercício apresentou correlação positiva e forte com $\operatorname{VEF}_{1}(\mathrm{r}=0,78)$ e fraca com escore do IMC ( $r=0,38)$ e inversamente proporcional ao tempo de hospitalização $(r=-0,42)$ e uso de antibióticos $(r=-$ 0,46) (Figura 1).

Quanto maior a porcentagem de tempo que os indivíduos passaram desenvolvendo atividades moderadas a vigorosas, maior o escore $Z$ do $\operatorname{IMC}(r=0,51)$ e menor o tempo de hospitalização $(r=-0,37)$. Assim como quanto maior a porcentagem de tempo em atividades sedentárias, menor o escore $Z$ do IMC ( $r=-0,43)$ (Figura2). Todas as correlações entre a capacidade de exercício e nível de atividade física com as variáveis clínicas estão expressas na Tabela 3.
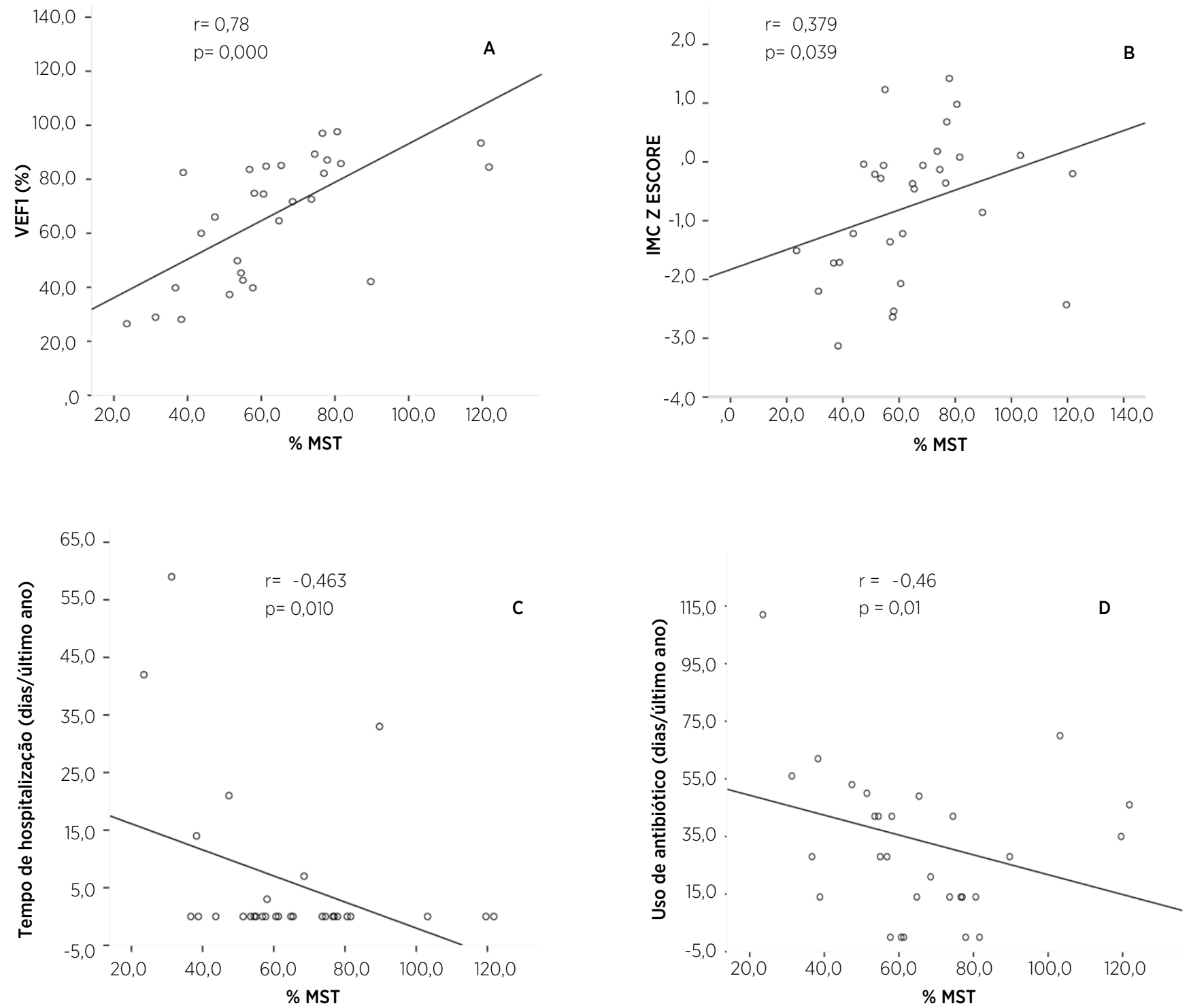

Figura 1. Correlação da capacidade do exercício com: (A) volume expirado forçado no primeiro segundo (VEF,\%); (B) escore Z do índice de massa corpórea (IMC); (C) tempo de hospitalização; e (D) uso de antibióticos 
Tabela 3. Correlação da capacidade de exercício e nível de atividade física com função pulmonar, estado nutricional, tempo de hospitalização de uso de antibióticos.

\begin{tabular}{|c|c|c|c|c|c|c|}
\hline & & \% Distância MST & Sedentário min/dia & \% Sedentário/dia & $\begin{array}{c}\text { Moderada e vigorosa } \\
\mathrm{min} / \mathrm{dia}\end{array}$ & $\begin{array}{c}\% \text { moderada e } \\
\text { vigorosa/dia }\end{array}$ \\
\hline \multirow{2}{*}{ Hospitalização (dias/ano) } & $r$ &,$- 423^{*}$ & ,272 & 279 &,- 341 &,$- 371^{*}$ \\
\hline & $p$ & ,020 & 147 & ,136 &, 065 & ,044 \\
\hline \multirow{2}{*}{ Antibiótico (dias/ano) } & $r$ &,$- 463^{*}$ &,- 108 &,- 038 &,- 058 &,- 072 \\
\hline & $p$ & ,010 &, 571 & 840 & ,760 & ,705 \\
\hline \multirow{2}{*}{$\operatorname{VEF}_{1}(\%)$} & r & ,782** & ,359 & ,339 & ,069 & ,076 \\
\hline & $\mathrm{p}$ & ,000 & 051 &, 067 & ,715 & ,688 \\
\hline \multirow{2}{*}{$\mathrm{FEF}_{25-75 \%}(\%)$} & r &, $665^{* *}$ &,- 255 &,- 305 &, $421^{*}$ &, $417^{*}$ \\
\hline & $p$ & ,000 & 175 & 101 & ,020 & ,022 \\
\hline \multirow{2}{*}{$\mathrm{IMC}(\mathrm{Kg} / \mathrm{m} 2)$} & $r$ & ,342 &, $415^{*}$ & ,450* &,- 169 &,- 152 \\
\hline & $\mathrm{p}$ & ,064 & ,023 & 013 &, 373 & ,424 \\
\hline \multirow{2}{*}{ Score Z IMC } & $r$ & ,379* &,$- 372^{*}$ &,$- 426^{*}$ &, $508^{* *}$ &, $511^{* *}$ \\
\hline & $p$ & ,039 & ,043 & ,019 & ,004 & ,004 \\
\hline
\end{tabular}

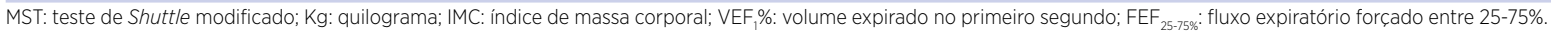

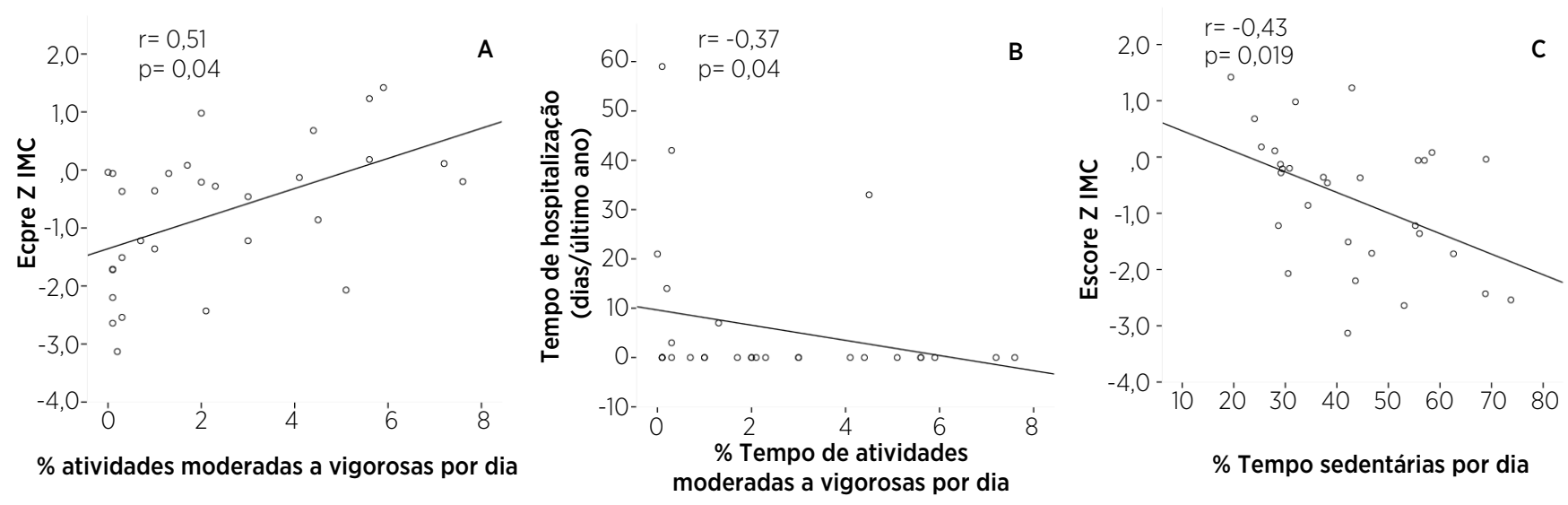

Figura 2. Correlação da \% de atividade física moderada a vigorosa diária com escore Z do índice de massa corpórea (A) e com dias de hospitalização (B). Correlação \% de atividade física sedentária diária e escore Z do índice de massa corpórea (C)

\section{DISCUSSÃO}

Nossos achados demonstram que mais da metade da amostra é sedentária, dispensando 354 minutos em atividades sedentárias - o que corresponde a $42 \%$ do tempo do dia - e apenas 14,9 minutos por dia realizando atividades moderadas ou vigorosas. O nível de atividade física, avaliado por aplicação de questionário, revelou que 64,6\% das crianças e adolescentes se autorreferiram como sedentários.

É preciso lembrar que a Organização Mundial da Saúde preconiza no mínimo 60 minutos de atividade física moderada a vigorosa por dia para crianças ${ }^{25}$ e que o sedentarismo é um problema de saúde pública e para a população estudada, pois leva à progressão da doença com comprometimento físico e funcional ${ }^{26}$. Estudos mostram que indivíduos com FC estão suscetíveis a quadro de desnutrição e inatividade ${ }^{3,27}$, ao analisar a correlação entre nível de atividade física e estado nutricional, encontramos correlação inversamente proporcional $(r=-0,46)$.
O exercício aeróbio melhora a aptidão cardiorrespiratória e muscular, sendo recomendado instituir atividades vigorosas, com consumo de sete METs para crianças e adolescentes, permitindo assim melhora da resistência cardiopulmonar, composição corporal, força muscular, resistência muscular, e flexibilidade ${ }^{28}$. Alguns autores demonstram que pacientes com fibrose cística frequentemente apresentam limitação do exercício físico, reduzindo progressivamente suas atividades de vida diária ${ }^{29,30}$. Uma recente revisão sistemática conduzida por Shelley et al. apontou associação positiva entre a capacidade de exercício e nível de atividade física mensurada por acelerômetro, entretanto o número de estudos é limitado e de baixa qualidade metodológica ${ }^{19}$. Nossos achados reforçaram essa associação, demonstrando que quanto maior o tempo em nível de atividade física moderada e vigorosa diária, melhor a tolerância ao exercício.

Saglam et al., em seu estudo em indivíduos com FC, função pulmonar normal e doença leve, relataram que o MST possui correlação moderada a forte com a função 
pulmonar $(\mathrm{p}<0,05)^{10}$, corroborando os achados deste estudo, no qual identificamos que valores reduzidos na espirometria são acompanhados por redução na distância percorrida no MST $(\mathrm{r}=0,69)$.

É importante que haja acompanhamento e orientação nutricional em pacientes com $\mathrm{FC}$, preconizando valores de IMC acima do percentil 50, que muitas vezes são difíceis de serem alcançados ${ }^{31}$. O estado nutricional exerce influência na função pulmonar, pois casos de desnutrição grave levam a piora importante na função pulmonar e aumento do risco de mortalidade ${ }^{32}$. Dados coletados prospectivamente em 319 crianças, mostraram que o ganho de peso de $1 \mathrm{~kg}$ está associado ao aumento no $\mathrm{VEF}_{1}$ em $32 \mathrm{~mL}$. Esses autores concluíram que as crianças com maior evolução de $\mathrm{VEF}_{1}$, eram aquelas que possuíam maior ganho de peso ${ }^{33}$.

Observamos correlação da capacidade de exercício e estado nutricional, em que valores maiores de IMC são acompanhados por uma maior distância percorrida no MST ( $r=0,39)$. Saglam et al. afirmam que o MST possui correlações moderadas-fortes semelhantes com idade, altura, peso $(\mathrm{p}<0,05)$, em que a medida de IMC contribuiu para a distância do MST $(\mathrm{p}<0,001)^{10}$. O presente estudo encontrou uma correlação moderada do MST com massa muscular esquelética $(r=0,52)$ e escore $Z$ do IMC $(r=0,43$, $p>0,05)$.

Em relação ao tempo de internação e uso de antibióticos em pacientes pediátricos com $\mathrm{FC}$, verificamos associação dessas variáveis com capacidade de exercício obtida na MST. Tanto o tempo de internação hospitalar $(r=-0,42)$ quanto o uso de antibióticos $(\mathrm{r}=-0,46)$ apresentam correlação moderada negativa com capacidade de exercício. A literatura tem mostrado que as variáveis levam ao prejuízo da capacidade funcional do indivíduo?.

Donadio et al. demonstraram que indivíduos com FC que andaram $<577,5$ metros tinham 4 vezes mais risco de serem hospitalizados e que a avaliação da capacidade funcional está associada ao menor risco de hospitalização no período de cinco anos ${ }^{9}$. Ainda, Waters et al. mostraram associação entre uso de antibióticos e declínio da função pulmonar em pacientes com fibrose cística, sugerindo que exarcebações pulmonares, tratadas com antibiótico intravenoso, podem ser um fator de risco para o declínio da função pulmonar ${ }^{8}$. Além disso, um outro estudo demonstrou que indivíduos com $\mathrm{VO}_{2}$ reduzida, obtido pela avaliação da capacidade de exercício, apresentam risco de mortalidade quase cinco vezes maior, indicando que pode ser um importante indicador prognóstico no acompanhamento das pessoas com fibrose cística ${ }^{14}$.

O presente estudo apresenta algumas limitações, incluindo, o desenho do tipo transversal, não nos permitindo avaliar de forma precisa a relação causaefeito. Além disso, não foi possível utilizar o teste de esforço cardiopulmonar, que é considerado o padrão ouro para avaliação da capacidade de exercício. No entanto, acreditamos que a utilização do MST, que já foi validado para uso em pacientes com $\mathrm{FC}$ e apresenta uma forte correlação com o $\mathrm{VO}_{2}$, foi adequada para avaliação da tolerância ao exercício na amostra estudada.

\section{CONCLUSÃO}

A capacidade de exercício em crianças e adolescentes apresentou-se reduzida e associada ao aumento do tempo de hospitalização, ao uso de antibiótico, à redução da função pulmonar e do estado nutricional. O tempo dispendido em atividade física de intensidade moderada e vigorosa por dia teve correlação com a distância caminhada no teste de Shuttle e foi associada a menor tempo de hospitalização e melhor estado nutricional, entretanto, o tempo que as crianças e adolescentes passam nesse tipo de atividade está muito abaixo do recomendado.

A avaliação da capacidade de exercício e a monitorização do nível de atividade física diária podem ser importantes preditores prognósticos em crianças e adolescentes com FC aliados a conhecidos indicadores como o $\mathrm{VEF}_{1}$ e ao estado nutricional, sendo ferramentas úteis no acompanhamento de rotina de pacientes com fibrose cística, direcionando a abordagem terapêutica multidisciplinar, com objetivo de reduzir os episódios de exacerbações e hospitalizações, melhorando prognóstico e qualidade de vida.

\section{REFERÊNCIAS}

1. Ribeiro MA, Ziegler B, Schivinski CIS, Aquino ES, Donadio MVF, et al. Recomendação Brasileira de Fisioterapia na Fibrose Cística: um guia das boas práticas clínicas. Assobrafir Ciencia. 2019;10(1):2160. [cited 2021 Aug 2]. Available from: https://bit.ly/3fpFrKM

2. Andrade Lima C, Andrade AD, Campos SL, Brandão DC, Mourato IP, Britto MCA. Six-minute walk test as a determinant of the functional capacity of children and adolescents with cystic fibrosis: a systematic review. Respir Med. 2018;137:83-8. doi: 10.1016/j. rmed.2018.02.016.

3. Hebestreit H, Arets HGM, Aurora P, Boas S, Cerny F, Hulzebos EHJ, Karila C, Lands LC, Lowman JD, Swisher A, et al. Statement on exercise testing in cystic fibrosis. Respiration. 2015;90(4):332-51. doi: 10.1159/000439057.

4. Rosa FR, Dias FG, Nobre LN, Morais HA. Fibrose cística: uma abordagem clínica e nutricional. Rev Nutr. 2008;21(6):725-37. doi: 10.1590/S1415-52732008000600011. 
5. Reid DW, Geddes LE, O'Brien K, Brooks D, Crowe J. Effects of inspiratory muscle training in cystic fibrosis: a systematic review. Clin Rehabil. 2008;22(10-11):1003-13. doi: 10.1177/0269215508090619.

6. Athanazio RA, Silva Filho LVRF, Vergara AA, Ribeiro AF, Riedi CA, Procianoy EFA, Adde FV, Reis FJC, Ribeiro JD, Torres LA, et al. Brazilian guidelines for the diagnosis and treatment of cystic fibrosis. J Bras Pneumol. 2017;43(3):219-45. doi: 10.1590/ S1806-37562017000000065.

7. Britto MT, Kotagal UR, Atherton HD, Wilmott RW, Hornung RW, Tsevat J. Impact of recent pulmonary exacerbations on quality of life in patients with cystic fibrosis. Chest. 2002;121(1):64-72. doi: 10.1378/chest.121.1.64.

8. Waters V, Stanojevic S, Atenafu EG, Lu A, Yau Y, Tullis E, Ratjen F. Effect of pulmonary exacerbations on long-term lung function decline in cystic fibrosis. Eur Respir J. 2012;40(1):61-6. doi: 10.1183/09031936.00159111.

9. Donadio MVF, Heinzmann-Filho JP, Vendrusculo FM, Frasson PXH, Marostica PJC. Six-minute walk test results predict risk of hospitalization for youths with cystic fibrosis: a 5-Year follow-up study. J Pediatr. 2017;182:204-9. doi: 10.1016/j.jpeds.2016.11.071.

10. Saglam M, Vardar-Yagli N, Savci S, Inal-Ince D, Aribas Z, BosnakGuclu M,et al. Six minute walk test versus incremental shuttle walk test in cystic fibrosis. Pediatr Int. 2016;58(9):887-93. doi: 10.1111/ ped.12919.

11. De Meer K, Gulmans VAM, van Der Laag J. Peripheral muscle weakness and exercise capacity in children with cystic fibrosis. Am J Respir Crit Care Med. 1999;159(3):748-54. doi: 10.1164/ ajrccm.159.3.9802112.

12. Schneiderman-Walker J, Pollock SL, Corey M, Wilkes DD, Canny GJ, Pedder L, Reisman JJ. A randomized controlled trial of a 3-year home exercise program in cystic fibrosis. J Pediatr. 2000;136(3):304-10. doi: 10.1067/mpd.2000.103408.

13. Ledger SJ, Owen E, Prasad SA, Goldman A, Willams J, Aurora P. A pilot outreach physiotherapy and dietetic quality improvement initiative reduces IV antibiotic requirements in children with moderate-severe cystic fibrosis. J Cyst Fibros. 2013;12(6):766-72. doi: 10.1016/j.jcf.2013.01.003

14. Vendrusculo FM, Heinzmann-Filho JP, Silva JS, Perez Ruiz M, Donadio MVF. Peak oxygen uptake and mortality in cystic fibrosis: Systematic review and meta-analysis. Respir Care. 2019;64(1):91-8. doi: 10.4187/respcare.06185.

15. Vallier JM, Rouissi M, Mely L, Gruet M. Physiological responses of the modified shuttle test in adults with cystic fibrosis. J Cardiopulm Rehabil Prev. 2016;36(4):288-92. doi: 10.1097/ HCR.0000000000000181.

16. Pulz C, Diniz RV, Alves ANF, Tebexreni AS, Carvalho AC, de Paola AAV, Almeida DR. Incremental shuttle and six-minute walking tests in the assessment of functional capacity in chronic heart failure. Can J Cardiol. 2008;24(2):131-5. doi: 10.1016/s0828-282x(08)70569-5.

17. Savi D, Simmonds N, Paolo MD, Quattrucci S, Palange P, Banya W, et al. Relationship between pulmonary exacerbations and daily physical activity in adults with cystic fibrosis. BMC Pulm Med. 2015;15:151. doi: 10.1186/s12890-015-0151-7.

18. Cox NS, Alison JA, Button BM, Wilson JW, Morton JM, Holland AE. Physical activity participation by adults with cystic fibrosis: an observational study. Respirol. 2016;21(3):511-8. doi: 10.1111/resp.12719.

19. Shelley J, Boddy LM, Knowles ZR, Stewart CE, Dawson EA. Physical activity and associations with clinical outcome measures in adults with cystic fibrosis; a systematic review. J Cyst Fibros. 2019;18(5):590-601. doi: 10.1016/j.jcf.2019.03.003.

20. Savi D, Paolo M Di, Simmonds N, Onorati P, Internullo M, Quattrucci $S$, et al. Relationship between daily physical activity and aerobic fitness in adults with cystic fibrosis. BMC Pulm Med. 2015;15:59. doi: 10.1186/s12890-015-0036-921.

21. Quanjer PH, Stanojevic S, Cole TJ, Baur X, Hall GL, Culver BH, Enright PL, Hankinson JL, Ip MSM, Zheng J, et al. Multi-ethnic reference values for spirometry for the 3-95-yr age range: the global lung function 2012 equations. Eur Respir J. 2012;40(6):132443. doi: 10.1183/09031936.00080312.

22. Lanza FC, Zagatto EP, Silva JC, Selman JPR, Imperator TBG, Zanatta DJM, Carvalho LN, Reimberg MMDS. Reference equation for the incremental shuttle walk test in children and adolescents. J Pediatr. 2015;167(5):1057-61. doi: 10.1016/j.jpeds.2015.07.068.

23. Kowalski KC, Crocker PRE, Columbia B, Donen RM. The Physical Activity Questionnaire for Older Children (PAQ-C) and Adolescents (PAQ-A) Manual. Saskatoon: University of Saskatchewan; 2004.

24. Evenson KR, Catellier DJ, Gill K, Ondrak KS, McMurray RG. Calibration of two objective measures of physical activity for children. J Sports Sci. 2008;26(14):1557-65. doi: 10.1080/02640410802334196.

25. World Health Organization. Global recommendations on physical activity for health. Geneva: WHO Library; 2010.

26. Walker RG, Obeid J, Nguyen T, Ploeger H, Proudfoot NA, Bos C, Chan AK, Pedder L, Issenman RM, Scheinemann K, et al. Sedentary time and screen-based sedentary behaviors of children with a chronic disease. Pediatr Exerc Sci. 2015;27(2):219-25. doi: 10.1123/ pes.2014-0074.

27. Santos RC, Mucha FC, Almeida ACS, Itaborahy BDH, Schivinski CIS. Força muscular respiratória e desempenho no Modified Shuttle Walk Test em escolares com fibrose cística. Fisioter Pesqui. 2019;26(2):196-201. doi: 10.1590/1809-2950/18045526022019.

28. Janssen I, Leblanc A. Systematic review of the health benefits of physical activity and fitness in school-aged children and youth. Int J Behav Nutr Phys Act. 2010;7:40. doi: 10.1186/1479-5868-7-40.

29. Chaves CRM, Britto JAA, Oliveira CQ, Gomes MM, Cunha ALP. Associação entre medidas do estado nutricional e a função pulmonar de crianças e adolescentes com fibrose cística. J Bras Pneumol. 2009;35(5):409-14. doi: 10.1590/ S1806-37132009000500004

30. Scalco JC, Martins R, Keil PMR, Mayer AF, Schivinski CIS. Psychometric properties of functional capacity tests in children and adolescents: Systematic review. Rev Paul Pediatr. 2018;36(4):50010. doi: 10.1590/1984-0462/;2018;36;4;00002.

31. Lusman S, Sullivan J. Nutrition and growth in cystic fibrosis. Pediatr Clin North America. 2016;63(4):661-78. doi: 10.1016/j. pcl.2016.04.005.

32. Turck D, Braegger CP, Colombo C, Declercq D, Morton A, Pancheva $R$, et al. ESPEN-ESPGHAN-ECFS Guidelines on nutrition care for infants, children, and adults with cystic fibrosis. Clin Nutr. 2016;35(3):557-77. doi: 10.1016/j.clnu.2016.03.004.

33. Peterson ML, Jacobs DR, Milla CE. Longitudinal changes in growth parameters are correlated with changes in pulmonary function in children with cystic fibrosis. Pediatrics. 2003;112(3.1):588-92. doi: 10.1542/peds.112.3.588. 